\title{
SEM Imaging of Resist Patterns Fabricated Through Imprint Lithography Techniques
}

\author{
C.T. Schamp, ${ }^{*}$ B.T. Valdez, ${ }^{*}$ L.K. Ballast,* A. Gill,* S.A. Pierce*, L. Brown**, C. Brooks, ${ }^{* *}$ E. \\ Thompson, ** B. Roth*** \\ * Cerium Labs, Austin, TX 78741 \\ ** Molecular Imprints, Austin, TX 78758 \\ *** Hitachi High Technologies of America, Pleasanton, CA 94588
}

Imprint lithography has the potential to continue the advancement in shrinking feature sizes beyond that of conventional photo-lithography. A special proprietary resist has been developed by Molecular Imprints which holds the promise of being the material of choice to define the incredibly small feature sizes required in future generation electronic devices. Like conventional photo-resists, this material charges under the electron beam at conventional SEM accelerating voltages, necessitating a study to find appropriate coatings or imaging conditions sufficient for metrology work of defined patterns.

Deposition of $\mathrm{Cr}$ through magnetron sputtering is a typical method for coating materials to reduce charging effects in SEM imaging. Magnetron sputtering involves ionizing a flowing gas, typically Ar, which is accelerated toward a target $(\mathrm{Cr})$ with enough energy to eject target atoms through the background gas and onto the sample. The higher the background gas pressure, the more likely there will be collisions with the ejected target atoms, leading to a more anisotropic deposition, but at a reduced rate. Experiments were carried out using a range of gas accelerating voltages (current settings), background gas pressures, and target materials. The experiments with Cr resulted in either deformation of the resist structures or implantation into the resist structures as seen in cross-sectional TEM micrographs shown in figure 1. Interestingly, AuPd - a heavier alloy than Cr, does not appear to deform the resist lines, but might be implanting, and the heaviest pure material used, Ir has been found to provide acceptable imaging results. These results will be shown in the presentation.

In series with the deposition experiments, imaging experiments were carried out with an Hitachi S4800 SEM using their patented E x B filter to selectively image with high-angle (HA) backscattered electrons (BS), or a combination of low-angle (LA) backscattered electrons and secondary electrons (SE) detected in the upper SE detector. The SE electrons originate closer to the surface and tend to reflect more of the topography of the surface and give edge artifact contrast, whereas the BS electrons tend to be higher energy and represent more of a Z-contrast of the material and are less sensitive to charging effects. High angle BS electrons tend to originate from the area of the sample surface at the location of the electron beam - where there is a single scattering event deflecting the beam electron through a nearly $180^{\circ}$ angle. Low angle BS electrons tend to have multiple scattering events within the sample and escape the sample surface farther from the entrance location with a lower angle relative to the beam direction. [1]

Typical metrology resist structures examined are parallel lines with a residual layer of resist material remaining between the lines. In top-down imaging of uncoated samples, the electron beam is interacting with only resist material, i.e. a single average molecular weight. To enhance contrast without charging artifacts, LA-BS electrons and SE electrons are combined forming a mixed image 
minimizing charging and edge artifact contrast. An example is shown in figure 2a. Cross-section images can be considered as imaging three separate phase spaces; the substrate, the resist, and vacuum. To enhance contrast between the different phases, HA-BS imaging is employed. The HA electrons provide good resolution as they originate from only the location of the beam, and do not suffer from the effects of charging as seen in figure $2 \mathrm{~b}$. To further enhance contrast, HA electrons can by filtered by using a longer working distance which reduces the entrance solid-angle of the HA electrons into the objective lens.

\section{References}

[1] Goldstein, et al., SEM and X-Ray Microanalysis, 3rd ed. Plenum press

[2] CTS would like to acknowledge Prof. David Joy for insightful comments during one of his presentations that motivated the non-conductive imaging experiments.
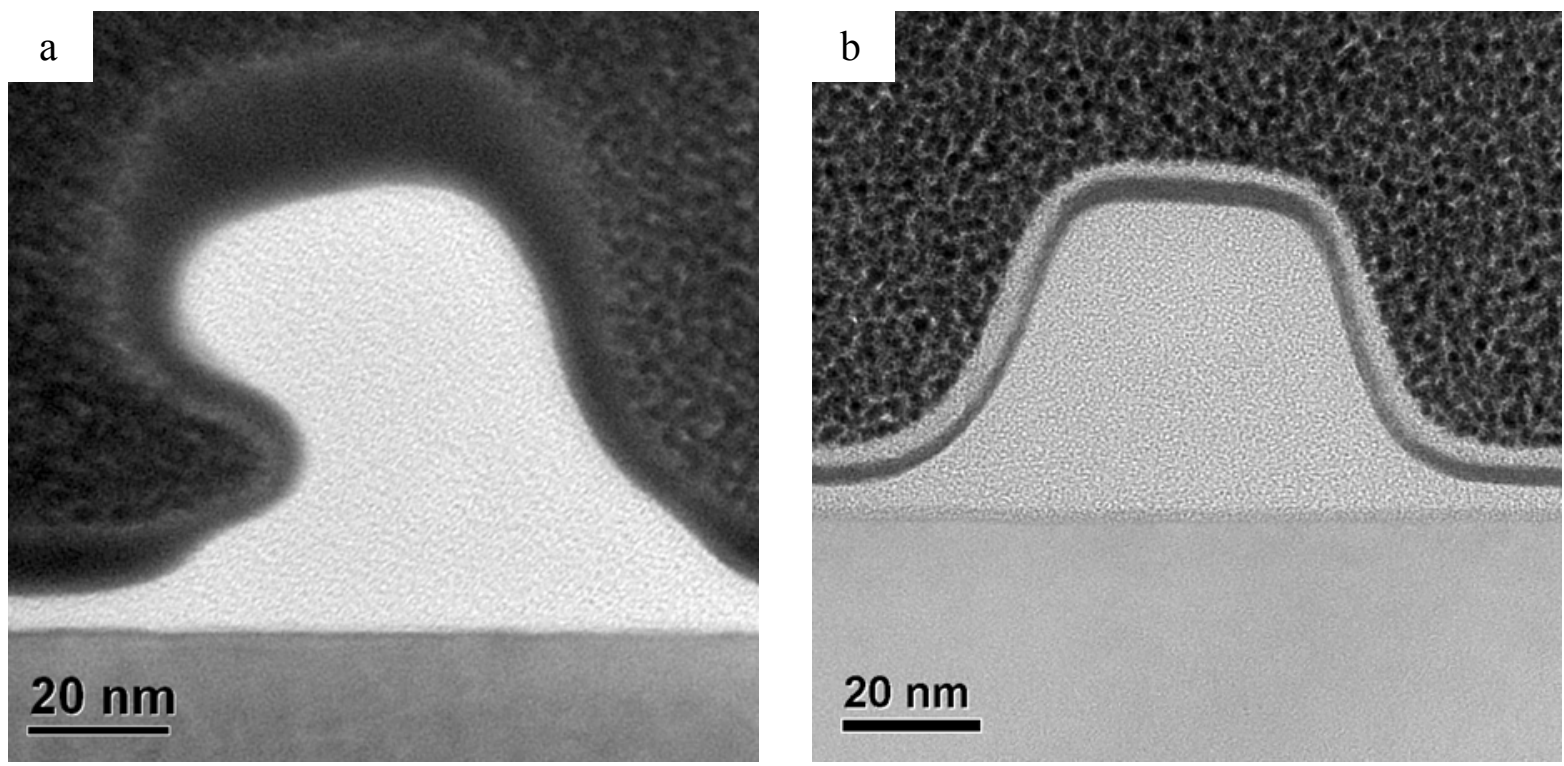

Figure 1. TEM micrographs of resist lines $\mathrm{Cr}$ coated through magnetron sputtering showing deformation in a) and implantation in b).
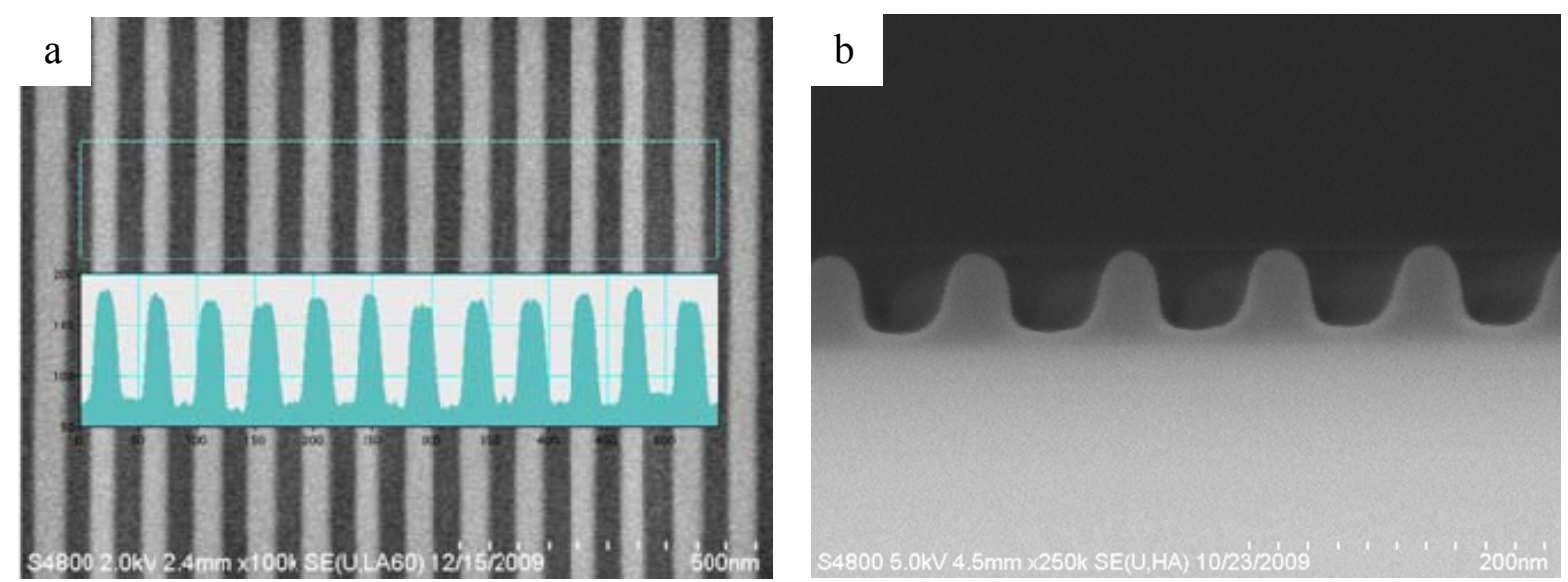

Figure 2. Top-down SEM imaging using a mixed signal of SE and LA-BS electrons producing an image without charging artifacts or edge contrast suitable for metrology in a), whereas cross-section SEM imaging using HA-BS electrons reduces charging artifacts while enhancing contrast between materials in $b$ ). 